\title{
BEHAVIOURAL ETIOLOGICAL FACTORS THAT CONTRIBUTE TO THE OCCURRENCE OF SEVERE EARLY CHILDHOOD CARIES
}

\author{
DANIELA EŞIAN ${ }^{1}$, CRISTINA BICA ${ }^{2}$, ANAMARIA BUD ${ }^{3}$, OANA STOICA ${ }^{4}$, \\ EUGEN BUD ${ }^{5}$ \\ 1,2,3,4, “George Emil Palade" University of Medicine, Pharmacy, Science and Technology, Tîrgu-Mures
}

\begin{abstract}
Keywords: severe early Abstract: Severe early childhood caries (S-ECC) is one of the most common pathologies affecting childhood caries, primary dentition. The complex etiology is the result of the interaction of microbial factors and also of breastfed, behavioural dietary factors. For this study a group of 40 children diagnosed with S-ECC was compared with a factors control group of 35 children without caries. The study data was obtained through clinical examination of both children and mothers, and on the basis of a questionnaire completed by the mothers of the subjects. The odontal status of the mothers was evaluated by determining the DMFT index. The results of the study showed that the mothers of the subjects with S-ECC had a significantly higher DMF-T caries index than the ones in the control group and that there is a close link between the birth of the child, newborn feeding method, mother's habits, cariogenic diet and the occurrence of severe early childhood caries.
\end{abstract}

\section{INTRODUCTION}

Severe-Early Childhood Caries (S-ECC) is currently a challenge for specialists and practitioners who frequently interact with small patients due to the early and aggressive onset immediately after the eruption of temporary teeth, but also to the rapid evolution of this type of caries that leads to a disturbance in the functional and aesthetic balance and thus an impairment of the little patient's quality of life. The complex and still incompletely elucidated etiology, is the result of the interaction of microbial factors represented in particular by S. mutans, which is the main microorganism responsible for the onset of the carious process which is transmitted during early childhood from mother to child, but also of dietary factors that are particularly related to the type of food intake, duration and method of administration (consumption habits). The following factors are involved in the development of severe early caries: pathogenic bacteria, fermentable carbohydrates and the quality of hard dental tissues. In addition to these three factors, incorrect eating behaviours associated with the absence of hygiene measures are also involved, leading to the rapid onset and evolution of S-ECC compared to other types of caries found in temporary dentition. $(1,2)$ Apart from S. mutans, S. sobrinus and Bifidobacteria also play an important role.(3)

In the absence of an early diagnosis and a complex treatment plan or even in the case of adopting late specific therapeutic measures, the difficulty of the treatment and also the costs involving a complex treatment can increase, and this is often a major obstacle for both doctors and parents. The first signs of caries appear at very young ages, shortly after dental eruption. When the pathogenic factors persist, the carious process can extend to all the erupted teeth at any given time, and it will have a tendency to rapidly evolve in surface and depth, with rapid crown destruction and early interest of the pulp tissue.(4) According to some authors the first lesions occur at the age of 20-22 months (5), while other authors report the occurrence of carious lesions at the age of 12-16 months (6) or even from the age of 10 months or earlier.(7) The localization of carious lesions depends on 3 factors: the chronology of the eruption of temporary teeth, the duration of bad habits and the muscle pattern of sucking. Thus, the first carious lesions occur in temporary superior incisors, but the carious process can extend to all erupted teeth at that time.(5) Expansion is done in the order of the eruption thus, the first affected group is of the upper central incisors, then the first molars, canines and finally, temporary second molars. If the lower incisors are not affected, the caries are called "bottle caries", but when they are affected, the caries are called "ramping caries".

In the evolution of S-ECC, 4 stages are described: the first stage, when incipient caries occur on the smooth surfaces of the temporary upper incisors in the 10-20 months old child. They occur in the form of white demineralization spots or an opaque or brown strip in the cervical third of the buccal and palatal surfaces of the upper incisors, parallel to the gingival margin.(2) In the second stage, which begins at the age of 16-24 months, cavitation lesions occur that destroy the enamel and reach the dentin, which becomes soft and changes in colour.(8) Stage three (at the age of 20-36 months) is characterized by the presence of deep carious lesions, extended in surface and with damage to the pulp tissue, in the upper incisors. Cavitation lesions occur in the upper molars and incipient caries occur on the first mandibular molars and the maxillary canines. The fourth stage, between 30-48 months, is characterized by massive destruction of the upper incisors, complicated caries occur on the superior molars, and deep simple caries occur in the inferior molars and superior canines.

Prevention of severe early childhood caries in

${ }^{4}$ Corresponding author: Oana Stoica, Str. Gheorghe Marinescu, Nr. 38, Târgu Mureș, România, E-mail: oana.stoica@umfst.ro, Phone: +40747 127603

Article received on 26.11.2020 and accepted for publication on 26.02.2021

AMT, vol. 26, no. 1, 2021, p. 70 


\section{CLINICAL ASPECTS}

temporary teeth should begin in the prenatal and perinatal period, with recommendations for mothers on the care of the infant and young child.(9) Pregnancy is an ideal period to promote ECC prevention given the profound influence of maternal oral health and behaviours on children's oral health.(10)

Secondary prophylaxis refers to the diagnosis and treatment of the caries process in the early stages (11) Complications resulting from severe early caries can have serious loco-regional and general consequences in the short or long term.(8) Thus, it was observed that within one year, children with carious lesions on their upper frontals had a mean dmfs index of the posterior teeth 2.5 times higher than those who did not initially have this type of caries.(12)

The consequences on the permanent teeth refer to the appearance of carious lesions, structure anomalies of local cause, the deviation of the eruption axis of the upper incisors and the reversal of the eruption order of the central incisors. The actual treatment of severe early childhood caries depends on the stage at which the carious process is detected but also on the degree of cooperation of the child. When the carious process is detected in the initial stages, the treatment involves stopping it's evolution by topical fluoride applications and restoring the existing carious lesions. If carious lesions are more extensive and pulp complications have occurred, an endodontic treatment is performed, specific to the stage of dental development, associated with a complex crown restoration with zirconium pedodontic crowns.

\section{AIM}

The purpose of our clinico-statistical study was to determine the role played by the prenatal and postnatal etiological factors in the occurrence of the severe early childhood caries S-ECC in a group of preschool children and their mothers.

\section{MATERIALS AND METHODS}

This study was carried out with the approval of the Local Ethics Committee of the Mureș Emergency Clinical County Hospital, in accordance with Decision No. 7843 of 28.05.2019.

For this study, a total of 75 children were examined, aged 1 to 3 years, with a mean age of 2.12 years. A main group consisting of 40 children diagnosed with S-ECC was selected and a control group consisting of 35 children without carious processes. In the study group 15 children were under 2 years of age and 25 children over 2 years of age. In the control group 21 children were under 2 years of age and 14 children over 2 years of age. The study data was obtained through clinical examination of both children and mothers, as well as on the basis of a questionnaire completed by the mothers of the children. All 75 children were examined in the presence of a parent, who was asked to sign an informed consent form before the consultation.

In the first stage, the subjects from the study group were clinically examined to confirm the diagnosis of S-ECC. The criteria for diagnosis of S-ECC according to American Academy of Pediatric Dentistry (AAPD) (13), are: in children younger than 3 years age any sign of smooth surface caries and over 3 years age one or more cavitated, missing or filled smooth surfaces in primary upper anterior teeth or filled score of $\geq 4$ (age 3), or $\geq 5$ (age 4), or $\geq 6$ (age 5).

In the second stage, a questionnaire was developed containing data on: age of the child, age of the mother at the time of birth, whether or not they consulted a dentist before birth, the type of diet and other aspects (table no. 1).

In the last stage of the study, the mother's clinical examination was performed and the DMF-T caries index was determined. During the determination of the DMF-T index, the following criteria were used:

each tooth was registered only once;

temporary restorations were considered carious lesions; a tooth was considered present, even though the crown part was completely destroyed and there was only a remaining root fragment.

The processing of the obtained data was done using the Microsoft Excel software - entering measured parameters, obtaining graphs, schematic representations through diagrams.

Table no. 1. Questionnaire addressed to the mothers of the subjects

QUESTIONNAIRE
Name, First name:
1. At what age did you give birth?
2. Was the birth natural or caesarean section?
3. Was the birth at term?
4. Have you consulted your dentist for pre-birth treatments?
5. How was the child fed: natural or artificial?
6. To what age has he been breastfed?
7. Did you usually feed your child at night (milk or tea)?
8. Did the child use to sleep with a bottle in his mouth?
9. Did you usually taste your child's food before feeding him?
10. Do you give your child natural juices or carbonated beverages?
11. At what age did the first carious lesions begin to appear in the child?
Affected teeth of the child:
Carious lesions of the mother:

\section{RESULTS}

Following the statistical processing of the data obtained from the questionnaire and from the clinical examination, the following results were obtained:

From the first questions it was observed that, $67.5 \%$ of mothers gave birth before the age of 30, the rest after 30 years and within the control group $60 \%$ of mothers gave birth before 30 years and the rest after the age of 30 .

To the question "Was the birth natural or caesarean section?", $75 \%$ of the mothers in the study group and $71.4 \%$ in the control group gave birth naturally, the rest being by caesarean section.

Regarding the question "Have you consulted your dentist for pre-birth treatment" it was observed that only $37.5 \%$ of the women in the study group attended a dental practice, while $60 \%$ of the women in the control group carried out a specialist appointment (figure no. 1).

Figure no. 1. Distribution of mothers of the subjects according to the pre-birth treatments

\section{Have you consulted your dentist for pre-}

\section{birth treatments?}

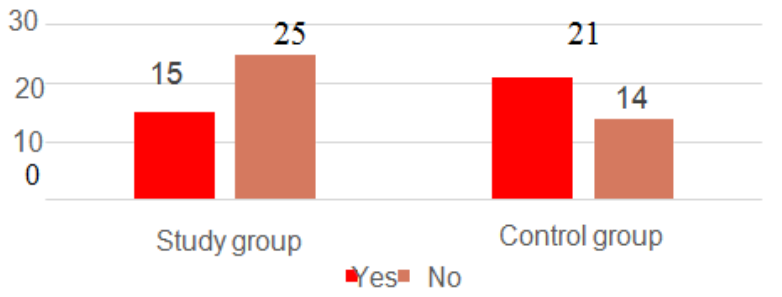

According to how the children were fed after birth the results show that $77.5 \%$ of the subjects in the study group and $60 \%$ of subjects in the control group were naturally fed (figure no. 2)

Regarding the eating habits, respectively "Did you 


\section{CLINICAL ASPECTS}

usually feed your child at night?", the results show that $97.5 \%$ of the subjects in the study group and $71.4 \%$ in the control group were fed at night.

Another question relates to the child's habit of sleeping with a bottle in his mouth, and the results obtained are presented in figure no. 3 .

Figure no. 2. Graphic representation of the subject's feeding behaviour after birth

\section{How was the child fed?}

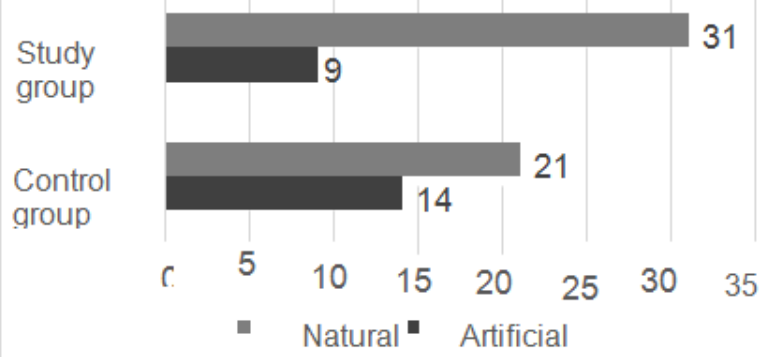

In terms of the bad habit of the child sleeping with the bottle in his mouth, only $65 \%$ of the subjects in the study group had this habit while in the control group the percentage is significant $(91.4 \%)$.

Figure no. 3. Graphic representation of the subjects according to eating habits

\section{Did the child use to sleep with a bottle in his mouth?}

40
30
20
10
0
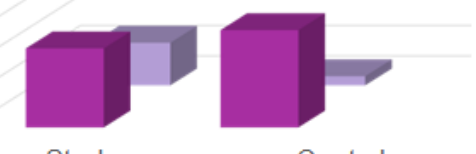

- Yes

Control group

No

Regarding the answers to the question "Do you give your child natural juices or carbonated beverages?", it was observed that in the study group these drinks were administered at a percentage of $47.5 \%$ and within the control group only $20 \%$ of the subjects received this type of drinks (figure no. 4).

In terms of age of occurrence of the first carious lesions it was observed that in a high percentage of subjects (77.5\%) from the study group the first carious lesions started after the age of 1 year.

Figure no. 4. Graphic representation of the consumption of carbonated beverages in subject groups

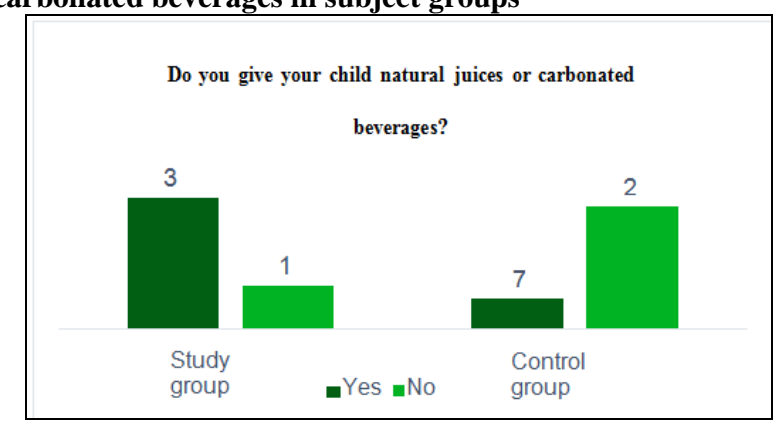

Another important factor involved in the occurrence of $\mathrm{S}-\mathrm{ECC}$ is the age up to which the baby was breastfed, so the data obtained from the questionnaire showed that $67.5 \%$ of the subjects in the study group were also breastfed after the age of 1 year while in the control group $28.5 \%$ exceeded the age of 1 year.

In the statistical data obtained from calculating the DMF-T caries of the mothers of the subjects in the study group, a mean index value of 4,575 was determined, while the mothers of the children in the control group had a mean value of 2.5 (figure no. 5).

Figure no. 5. The mean value of the DMF-T index of the mothers in both study and control groups

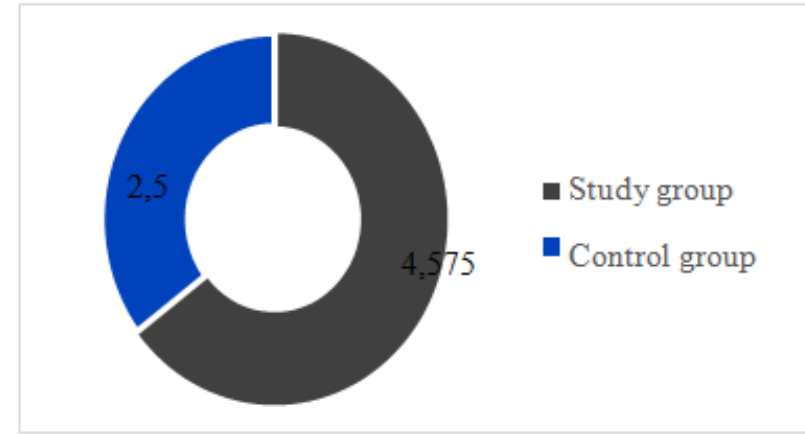

\section{DISCUSSIONS}

The correlation between the occurrence of severe early childhood caries and childbirth, the eating behaviour and the habits of the mother, is a widely debated topic in the literature. Various clinical studies conducted in other countries, focused on the consumption of cariogenic foods and the presence of bacteria have indicated a statistically significant correlation between these elements. Thus, a study conducted in the USA on 110 children, of which 72 children had severe carious lesions, out of all subjects it was observed that the ones affected by recurrent caries had higher scores in the consumption of carbonated drinks between meals and also the meals were more common than in children who did not have carious lesions. Among the bacteria, those associated with the occurrence of recurrent lesions are S. mutans and S. sobrinus. Children with S. mutans had higher food cariogenicity scores. The conclusion of this study was that frequent meals, presumed cariogenicity and S. mutans are associated with the presence of severe early childhood caries.(14)

Our study revealed an increased percentage of S-ECC occurrence in children who used to consume carbonated beverages.

Regarding the importance of breast-feeding, additional sugary food or pre-chewed mother food, a study carried out in Southeast Asia among mothers with children aged 25 to 30 months, in a community where prolonged breastfeeding is a common practice, it was observed that all children who consumed additional sugary food or rice pre-chewed by the mother or children who fell asleep with the breast in their mouth, had lesions specific to severe early caries. The infants without these habits, who were breastfed up to 12 months had no carious lesions. The conclusion of this study was that in this population, in addition to the sugar and pre-chewed rice consumption, nocturnal breastfeeding after the age of 12 months is a risk factor in the development of severe early childhood caries.(12)

In the present study, it was observed that the age to which the child was breastfed is an important factor in the occurrence of S-ECC. The obtained data showed that $67.5 \%$ of 


\section{CLINICAL ASPECTS}

the children in the study group and $28.5 \%$ of the children in the control group were breastfed for more than one year.

In 2017, in Moradabad, India, the association between maternal risk factors and early severe caries was studied among children aged 3 to 5 years. The study involved 150 child-mother pairs. The maternal risk factors were assessed using a questionnaire. After obtaining consent, the mothers and children were clinically examined for caries using the Radike criteria (1968). Saliva was collected from all participating mothers in order to assess the level of Streptococcus mutans. The obtained data showed significant differences between the carious activity of the mothers, the high level of Streptococcus mutans, the educational level, the socio-economic status, the frequency of maternal sugar consumption and caries incidence in children. 15 In our study, the results show that the mothers of the children in the study group had a significantly higher DMF-T caries index $(4,575)$ than the mothers of the children in the control group(2.5).

Another study conducted in the city of Xiamen in China, made the correlation between social and behavioural factors in the occurrence of S-ECC. The study was carried out on 1523 children with a prevalence of severe early childhood caries of $56.8 \%-78.31 \%$. The factors associated with this pattern of caries were: age, consumption of sweets, carbonated drinks, eating before bedtime, late start of oral hygiene, low parental education, flawed care of children, increased number of siblings, rural residence and lack of knowledge of oral health. The study concluded that tooth brushing and topical application of fluoride are two effective methods of preventing caries. The results showed that the brushing frequency and type of toothpaste are not significant, but the earlier start time of brushing and topical application of fluoride is significantly associated with decreased incidence of caries.(16)

In Salvador, Bahia the association between S-ECC and low birth weight was studied. The examined independent variables were socio-demographic, perinatal and behavioural, such as the type of breastfeeding. The survival analysis was used to estimate the development period of the caries using the Kaplan-Meier method. Rural or urban environment, daily selfcare habits, age, skin colour, maternal education, family income, biofilm presence and subsequent experience with caries were the determining factors until the occurrence of caries. A positive association was found between low birth weight and the period for the development of caries. In conclusion, low birth weight is associated with the appearance of carious lesions.(17)

The relationship between severe early childhood caries and salivary alpha amylase, carbonic anhydrase VI and the presence of biofilm was shown in a study conducted by Brazil. The purpose of the study was to determine whether these factors can predict the risk for caries. 100 children were examined, who were divided into two groups: 45 children with carious lesions and 55 children without carious lesions. The presence of biofilm on the maxillary incisors was recorded, followed by the diagnosis of caries at the initial time and later. Saliva samples were collected and the activities of carbonic anhydrase VI and alpha amylase were determined. The normality of the data was assessed using the Shapiro-Wilk test. The results showed that the activity of carbonic anhydrase VI was higher in children with caries, and the activity of alpha amylase was significantly higher in the saliva of children without cavities. Children with alpha amylase activity in saliva less than $122.8 \mathrm{U} / \mathrm{mL}$ and visible presence of biofilm on the superior incisors are more prone to developing of severe early childhood caries. In conclusion, the visible presence of biofilm and the decreased activity of alpha amylase can be considered risk factors in the development of severe early childhood caries.(18)
In our study, another important factor related to the occurrence of S-ECC is night-time feeding. Thus, it was concluded that in the study group, there were $26 \%$ more breastfed children during night-time than in the control group.

\section{CONCLUSIONS}

The results of the studies show that there is a close link between the birth of the child, the newborn feeding method (natural or artificial), mother's habits (pre-chewing the food of the child), the cariogenic diet (sweetened drinks, sweet foods) and the occurrence of severe early childhood caries. Significant differences were found between the mother's caries index, high level of Streptococcus mutans, educational level, socioeconomical status, frequency of maternal sugar consumption and the onset of severe early childhood caries.

There was also a positive association between the consumption of carbonated beverages and the development of this type of caries. The child's night-time feeding, the breastfeeding that extends beyond the age of one year and the mother's DMF-T caries index, also play an important role in the occurrence of S-ECC, and they all indicate high values in the study group.

\section{REFERENCES}

1. Luca R, Ivan A, Stanciu I, V A. Severe early childhood caries in a sample of preschool children attending a Pediatric Dentistry Clinic from Bucharest. Oral Health Dent Manag. 2002;1: 29-35.

2. Attari N, Roberts JF. Restauration of primary teeth affected by Early Childhood Caries. Eur J Paed Dent.2004;2: 92-7.

3. O'Sullivan DM, Tinanoff N. Social and biological factors contributing to caries of the maxillary anterior teeth. Pediatr Dent. 1993;15(1):41-4.

4. Tinanoff N, Kanellis MJ, Vargas CM. Current Understanding of the epidemiology, mechanism and prevention of dental caries in preschool children. Pediatr Dent. 2002;24(6): 543-51.

5. Luca R. Pedodonție, Vol. 2, Ed. Cerma, București; 2003. p 49-70.

6. Ramos FJ, Weintraub JA, Gansky, H CI, F JDB. Bacterial, behavioral and environmental factors associated with early childhood caries, Pediatr Dent. 2002;26(2):165-73.

7. Anil S, Anand PS. Early Childhood Caries: Prevalence, Risk Factors, and Prevention. Front Pediatr. 2017;5:157.

8. Aneta M, Luca R. Caria severă precoce la dinții temporari, Ed. Eurobit, Timișoara; 2014.

9. Ismail AI, Sohn W. A sistematic review of clinical diagnostic criteria of early childhood caries. J Public Health Dent. 1999;59(3):171-91.

10. Xiao J, Alkhers N, Kopycko-Kedzierawski DT, Billings RJ er al. Prenatal Oral Health Care and Early Childhood Caries Prevention: A systematic review and Meta-Analysis. Caries Res. 2019;53(4):411-421.

11. Oltean D, Pătroi G, Cuculescu M. Stomatologie preventivă, Ed. Anotimp; 1996.p. 7-12,47-50, 61-140.

12. WH van Palenstein Helderman, Soe W, van 't Hof MA. Risk factors of early childhood caries in a Southeast Asian population. J Dent Res. 2006;85(1):85-88.

13. American Association of Paediatric Dentistry, Policy on Early Childhood caries: Classification, Consequences, Preventive Strategies.Oral Health Policies. 2011;33(6):4749.

14. Palmer CA, Kent R Jr, Loo CY, Hughes CV, Stutius E, Pradhan N, Dahlan M, Kanasi E, Arevalo Vasquez SS, Tanner AC. Diet and caries-associated bacteria in severe early childhood caries. J Dent Res. 2010;89(11):1224-9.

15. Agarwal V, Nagarajappa R, Keshavappa SB, Lingesha RT. 
Association of maternal risk factors with early childhood caries in schoolchildren of Moradabad, India. Int J Paediatr Dent. 2011;21(5):382-8.

16. Li Y, Zhang Y, Yang R, Zhang Q, Zou J, Kang D. Associations of social and behavioural factors with early childhood caries in Xiamen city in China. Int $\mathrm{J}$ Paediatr Dent. 2011;21(2):103-11.

17. da Silva Castro CR, de Sousa Cabral MBB, Mota ELA, Cangussu MCT, Vianna MIP. Analysis of the influence of low birth weight on the time of eruption of dental caries in children in early childhood. J Public Health Dent. 2019;79(4):292-297.

18. Gabriela N, Livia P, Lenita L. Relationship among alfa amylase and carbonic anhydrase VI in saliva, visible biofilm and early childhood caries: a longitudinal study. Int J Paedr Dent. 2016;27(3):174-182. 